\title{
Network Representation of User's Mechanical Impedance as Design and Evaluation Basis for Task-Specific Haptic Systems
}

\author{
Christian Hatzfeld ${ }^{1}$, Thorsten A. Kern ${ }^{2}$, Carsten Neupert ${ }^{1}$, Jan Lotichius ${ }^{1}$, Roland Werthschützky ${ }^{1}$ \\ ${ }^{1}$ Technische Universität Darmstadt, Institut für Elektromechanische Konstruktionen, \\ Merckstr. 25, 64283 Darmstadt, Germany. c.hatzfeld@emk.tu-darmstadt.de \\ ${ }^{2}$ Continental Corporation, VDO-Str. 1, 64832 Babenhausen, Germany
}

\begin{abstract}
This paper deals with the representation of the human user of a haptic system in the design process and investigates different fitting algorithms to find network representations and transfer functions. The user, represented by the mechanical impedance, serves as mechanical load to the system and influences the assessment of the quality of the haptic feedback. Due to large inter-personal variances, the mapping from measurements to concentrated network parameters is investigated in this paper.

Three different fitting approaches are used and compared based on the model error. The results show lowest errors for fitting algorithms that incorporate both amplitude and phase information of the measurements despite a linearity assumption of the basic network model. Suggestions for the mapping of the transfer functions to parameter values of the network model are given.
\end{abstract}

Key words: haptic system design, network representation, user impedance, vector fitting, system identification

\section{Motivation}

Haptic systems address the human sense of touch to convey information, either additional to visual and auditory information already present or as a new form of interaction. One of the major application areas in research and industry is adding touch information to medical and surgical systems to increase task performance and patient's security. Such procedures like heart catheterization [1] and laparoscopic interventions [2] exhibit quite special interaction schemes. Therefore more and more task-specific haptic systems are designed to add touch information and force feedback.

Such systems convey forces and vibrations in several degrees of freedom. For this purpose, manifold actuator principles and kinematic structures are used $[3,4]$ for operating devices and manipulator systems. Depending on the kinematic mechanisms used and the selected actuator principles, operating point dependent and multi-dimensional transfer functions arise already for a single element of a haptic system.

Model-based evaluation of system designs regarding the control stability and the quality of the delivered haptic feedback is necessary for the optimization of both criteria. Modeling techniques used for this purpose therefore have to be able to reproduce the above mentioned properties of systems, while allowing computationally efficient calculations. For the electromechanical systems, network theory based on lumped or distributed parameters seems to be an attractive modeling option [5]. It has been shown, that network-based models can be used to model and optimize haptic operating devices $[3,6]$, as well as the mechanical properties of interaction environments.

Since a haptic system will interact with a human user, this paper addresses the representation of the user in system design models. In the next sections, the general design process of haptic systems is briefly outlined and two modeling options are discussed. Section 3 focuses on the importance of the user and its influence on stability and haptic quality. Section 4 then presents measuring and modeling techniques for user impedances.

\section{Design of Haptic Systems}

Haptic (teleoperation-)systems consist out of three principal system components:

- Haptic operating device: Delivers haptic feedback to the user and records control signals for the manipulator. 
- Manipulator system: Interacts with the environment and records reaction forces that are presented as haptic feedback to the user. Typical manipulator systems include universal industrial robots or virtual environments that are for example used for surgical training purposes.

- System controller: To ensure stability regardless of operating points, system latency and user reactions, the system controller is a crucial part of a haptic system. Besides stability, the quality of haptic feedback can be influenced by the system controller.

In general, a haptic operating device consists out of several actuators (at least one for each actuated degree of freedom), a kinematic mechanism and several sensors to record the user input. For a high quality feedback, additional sensors are used to close a control loop for the force or position output of the device. If a real manipulator system is used instead a virtual environment, this system exhibits a similar structure with actuators, kinematic mechanisms, sensors and internal control loops. To make up a complete haptic system, the system controller closes two additional control loops for control signals from the haptic operating device to the manipulator and for measured signals from the manipulator to the haptic operating device.

To model such systems, two different approaches are commonly used. The first approach uses transfer functions linking different input $x_{\text {in }}(s)$ and output $x_{\text {out }}(s)$ signals of an arbitrary system component by a frequency dependent complex function according to eq. 1 .

$$
G(s)=\frac{x_{\text {out }}(s)}{x_{\text {in }}(s)}
$$

The physical representation of the signals $x_{\text {in }}$ and $x_{\text {out }}$ depend on the component described by the transfer function $G$. If $G$ describes a common DC motor, $x_{\text {in }}$ could describe the voltage applied to the motor and $x_{\text {out }}$ would describe the rotational speed. Another transfer function would link coil current $i$ with torque $M$. Transfer functions can be linked and the overall transfer function can be calculated by multiplying the linked transfer functions

The second approach is the use of concentrated network parameters and two-portrepresentations of the systems components. The network uses two independent coordinates, that model the flux between two connected nodes in the network (flux coordinate $f$ ) and the potential difference between two arbitrary nodes (not necessarily directly connected, differential coordinate $d$ ). Coordinates are chosen depending on the represented domain, i.e. electrical, mechanical or thermal systems. In general, the product of flux and differential coordinates resembles power. For electrical systems, voltage $u$ is chosen as differential coordinate, current $i$ serves as the flux coordinate. System components are modeled with networks of of basic lumped elements like resistors, capacitors and inductors (electrical domain) or masses, viscous dampers and springs (translational mechanical system).

Compared to the description of transfer functions, each system component is represented by two input and two output signals, In this case, the above mentioned motor is described by a complex matrix $H$ linking mechanical and electrical properties as given in eq. 2 .

$$
\left(\begin{array}{l}
\Omega(s) \\
M(s)
\end{array}\right)=\left(\begin{array}{ll}
h 11 & h 12 \\
h 21 & h 22
\end{array}\right)\left(\begin{array}{l}
u(s) \\
i(s)
\end{array}\right)
$$

Obviously, eq. (2) incorporates eq. (1), but extends the motor model in such a way, that arbitrary electrical and mechanical boundary conditions can be considered. Despite specific two-port-models like eq. 2, general transducers are known to link different domains in a network representation of a system.

Comparing both modeling options, the network representation proves to be more illustrative, since each network component has a physical representation. Since the connection between different system components includes differential and flux components (and therefore also models energy flux), components are independently interchangeable without a recalculation of transfer functions. Calculation of system models based on network parameters are fast and efficient, since proven calculation tools like SPICE can be used. On the contrary, network models are restricted to systems with a linear behavior. This is normally accounted for by calculating a working point and considering only small changes.

\section{Considering the user}

Regarding the design of haptic systems, the user plays two important roles. First, the user is the standard for the quality of haptic feedback. Recent studies of the authors show, that haptic transparency is closely connected to the mechanical impedance of the user $\underline{z}_{\text {user }}$ in the given contact situation. Second, the user comprises a mechanical load to the operating device. According to [7], the user can be represented as a combination of an active source of forces $\underline{F}$ or velocities $\underline{v}$ and a passive 
mechanical load, i.e. the mechanical impedance $z$ as given in eq. 3 .

$$
z(s)=\frac{F(s)}{v(s)}
$$

The knowledge of the mechanical user impedance is therefore an important prerequisite in the design of haptic interfaces. To describe this mechanical impedance, [8] proposes a second order spring-mass-damper model. This simple model is effective in calculating forces for certain contact situations, but cannot incorporate more complex dependencies encountered in the design process. Based on $[9,10]$, one of the authors therefore proposes a more complex model based on network parameters. This model is capable to incorporate external parameter like contact force, age and temperature [11] by selectively altering elements of the network model [4]. The network representation is given in fig. 1.

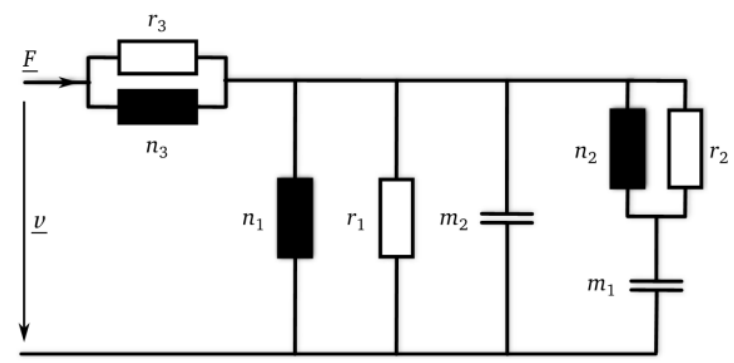

Fig. 1: Network representation of the mechanical impedance $\underline{z}_{u s e r}$ using masses $(m)$, springs (n) and dampers (r).

In the model, $r_{3}$ and $n_{3}$ describe the mechanical properties of the skin in direct contact with the haptic system, while $n_{1}, r_{1}$ and $m_{2}$ describe the properties of the directly attached limb, for example the finger. With $n_{2}, r_{2}$ and $m_{1}$, properties of farther body parts like joints and limbs are described. While the model is able to incorporate external parameter, nonlinear behavior like relaxation cannot be described thoroughly by this model. This is tolerable in respect to the above outlined design of haptic systems, since the general network representation cannot describe such components as well. Because of the all-springconnection, the model is not defined for frequencies $\omega \rightarrow 0$; this has to be taken into account when selecting fitting.

Measurements show a large inter-personal variance of the mechanical impedance for different users. Therefore, robust and accurate fitting methods for measurement data in the above described mode are needed. In the following, the used measurement method for the mechanical impedance $\underline{z}_{\text {user }}$ is described and several fitting algorithms to obtain network parameters in fig. 1 are evaluated.

\section{Measuring and Modeling Mechanical Impedances}

\subsection{Measurement Setup and Results}

The measurement setup used consists of a force source (Brüel \& Kjear 4810) that provides a sinusoidal force with a peak value of $10 \mathrm{~N}$ in a frequency range of $\mathrm{DC}$ to $18 \mathrm{kHz}$. For measuring the mechanical Impedance an impedance sensor (Brüel \& Kjaer model 8001, operating frequency up to $10 \mathrm{kHz}$ ) with integrated piezoelectric force and acceleration sensor is used. The speed response $v$, necessary to compute the mechanical impedance, defined in eq. (3), is obtained by integrating the measured acceleration signal. To provide the force signal and to compute the mechanical impedance a network analyzer (model Agilent $35670 \mathrm{~A}, f_{\text {sample }}=51,2 \mathrm{kHz}$ for each channel) is used.

During the measurement the sinusoidal force is exposed to the fingertip under test. To couple the force to the finger, a round concave plate with a diameter of $19 \mathrm{~mm}$, well adapted to the finger to create a large contact area, is used as interface. The measurement setup is shown in fig. 2 .

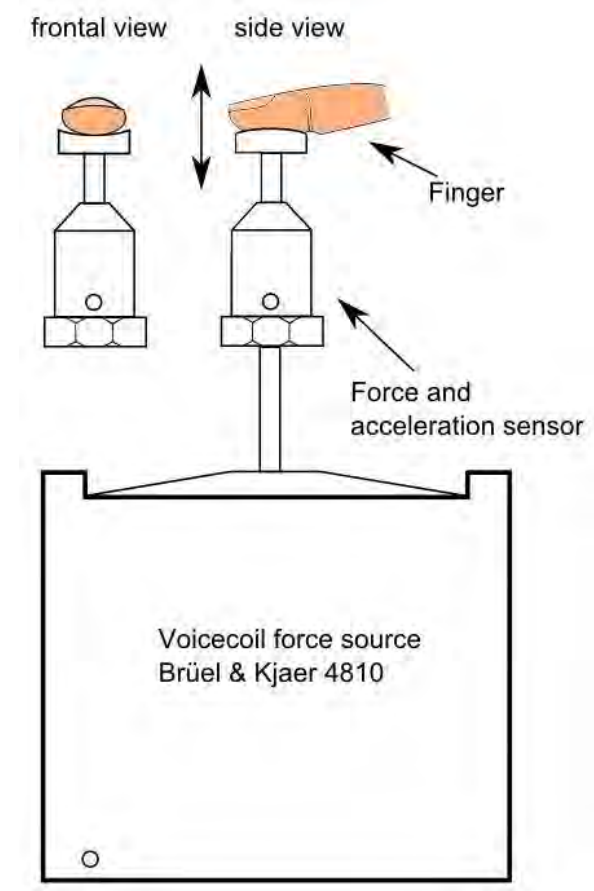

Fig. 2: Measurement Setup. The contactor used is made from aluminum with a diameter of $19 \mathrm{~mm}$ and a concave cut out with a radius of $31 \mathrm{~mm}$ along the finger axis.

Each measurement is done in a frequency range of $5 \mathrm{~Hz}$ to $5000 \mathrm{~Hz}$ with 401 

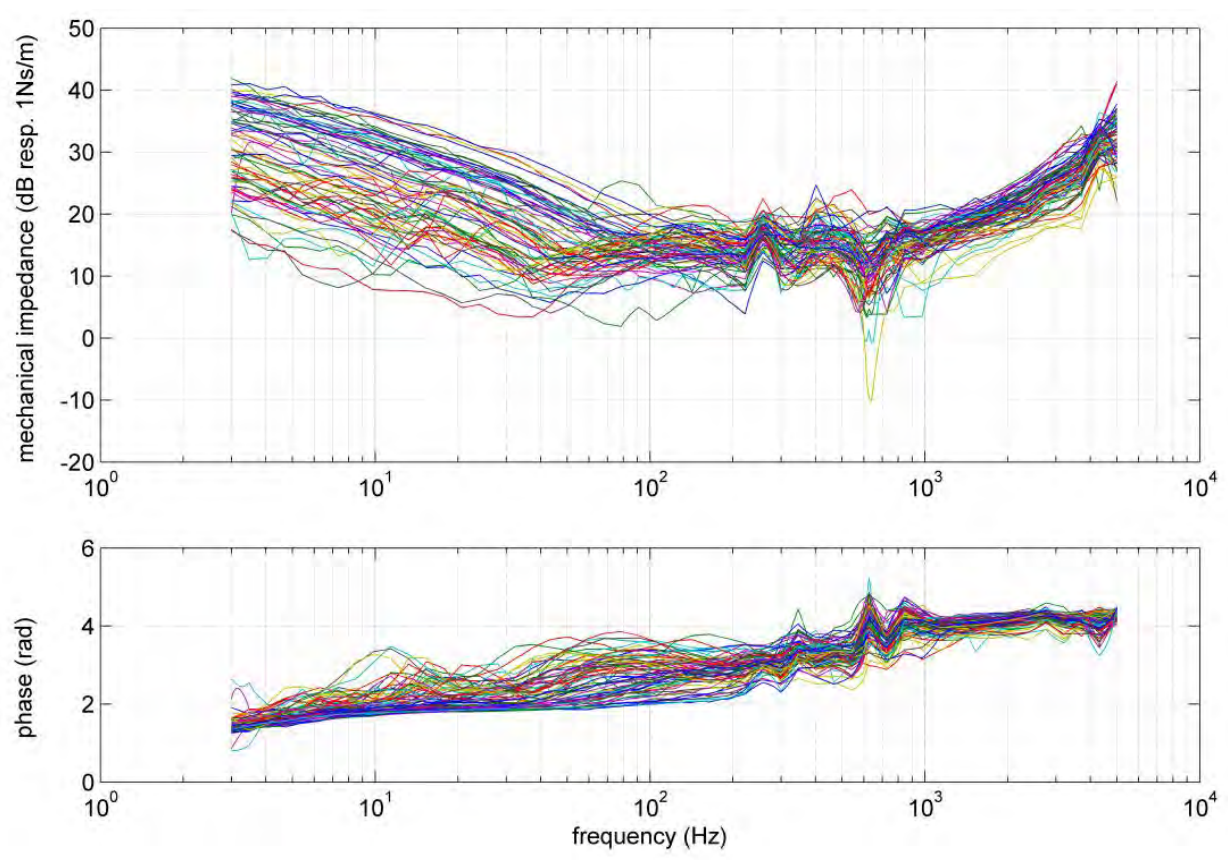

Fig. 3 Measurements of the mechanical impedance $z_{\text {user }}$ of $n=71$ test persons.

logarithmically distributed sample points. To reduce measurement uncertainties the measured value of each sample is taken as the mean of 5 periods, after waiting 5 periods to create a steady state. Furthermore the average of three measurements of each person under test was used for further calculations and fits.

To produce comparable results the test persons were asked to provide a constant offset force to the measurement setup with a value of $1 \mathrm{~N}$. The offset force was measured and fed back with an oscilloscope where, in addition to the current offset force, a tolerance band of $\pm 10 \%$ was displayed. The produced offset force of each subject was in the tolerance band.

To eliminate the characteristics of the measurement setup a calibration measurement is done without any load on the finger interface and afterwards subtracted from the measured impedance values. The results of the measurements are given in fig. 3 .

While the general shape of the curves is similar with decay up to a frequency of $100 \mathrm{~Hz}$, resonance effects between $200 \mathrm{~Hz}$ and $1 \mathrm{kHz}$ and an ascending slope above that, a large interpersonal variance can be seen. Reasons could be different skin conditions (moisture, calloused skin) and erroneous user behavior during the measurement process. When each measured frequency point is analyzed separately, over $75 \%$ of the impedance values are normal distributed ( $X^{2}$-Test, $\alpha=0,05$, amplitude and phase analyzed separately), therefore a central value and dispersion parameter for the network elements given in fig. 1 should describe the users impedance sufficiently.

\subsection{Modeling Methods}

Based on the linearity assumption of the model in fig. 1, all data sets were fitted to the transfer function given in eq. (4) using MATLAB with a Levenberg-Marquardt-algorithm. Absolute amplitudes and phases were fitted separately and mean and median of each network element were computed. A model transfer function $Z_{\text {model }}$ was calculated with these values and the average quadratic error for the amplitude according to eq. (5)

$\Delta_{\mathrm{m}, \mathrm{A}}(f)=\frac{1}{n} \sum_{i=1}^{n=71} \sqrt{\left(\left|\underline{z}_{\text {model }}(s)\right|-\left|\underline{z}_{i}(s)\right|\right)^{2}}$

was calculated for each frequency as a measure of the fit quality. A similar defined error $\Delta_{\mathrm{m}, \mathrm{P}}$ was calculated for the phase information. As a reference, the mean and the median of the measured impedances were considered.

The analysis of this error is given in fig. 4 as average of the errors $\Delta_{\mathrm{m}, \mathrm{A}}$ and $\Delta_{\mathrm{m}, \mathrm{P}}$ and the corresponding standard deviation. It is obvious that the assumption of linearity does not hold, since models based on amplitude fits exhibit

$$
\underline{z}_{u s e r}(s)=\left(\frac{s n_{3}}{s r_{2} n_{3}+1}+\left(\frac{-s^{2} m_{2} n_{1}+s n_{1} r_{1}+1}{s n_{1}}+\left(\frac{1}{s m_{1}}+\frac{s n_{2}}{s n_{2} r_{2}+1}\right)^{-1}\right)^{-1}\right)^{-1}
$$




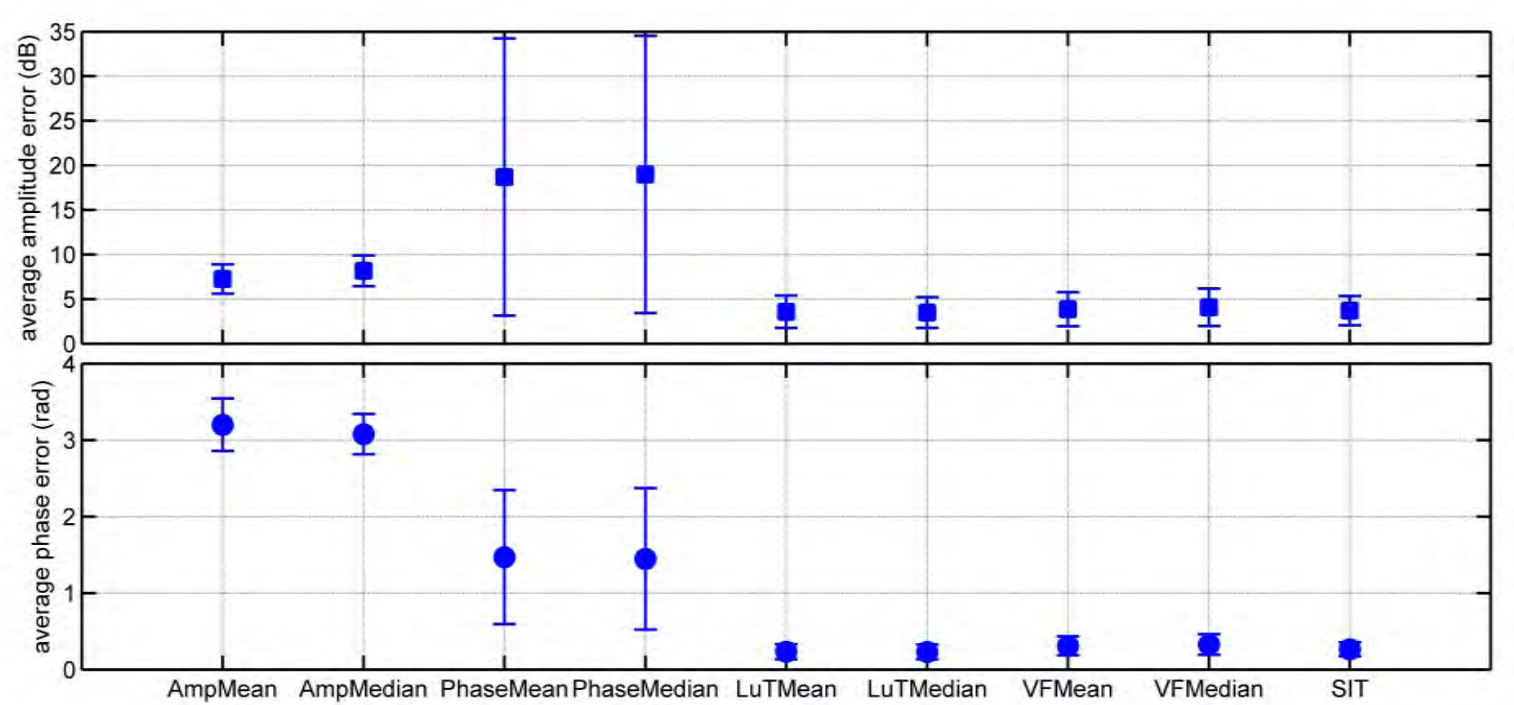

Fig. 4 Comparison of $\Delta_{m, A}$ and $\Delta_{m, P}$. Shown is the average error for each tested person and the corresponding standard deviations over all considered frequencies. Mean and Median denote the statistical parameter used. First two data sets are based on models based on amplitude fitting, the second two data sets are based on fitting of the phase. LuT denotes Look-up-Table implementation, VF Vector Fitting, considering amplitude and phase values simultaneously. SIT denotes the usage of the system identification toolbox of MATLAB.

large phase errors and vice versa. In general, all fits were worse than the reference implementations of mean and median. This leads to the conclusion, that the model given in fig: 1 and eq. (2) is not sufficient in representing the real features and has to be further extended.

Two approaches are evaluated to find a suitable expression for the network parameters of the model in fig. 1 with a low error. The first approach is the use of standard system identification tools (SIT) that estimate poles and zeros of the measurements and minimize errors by non-linear least-square searches (MATLAB function tfest with standard parameters and eq. (4) as a model). Eq. (2) is used as a functional basis for this algorithm. The second approach is the vector fitting method [12-14]. This method estimates the poles of the transfer function with an intermediate model and calculates the real poles of the unknown transfer function by optimizing this intermediate model. This method was investigated based on the mean (VFMean) and the median (VFMedian) of the measurement data.

As shown in fig. 4, both models yield errors in the range of the errors of the lookup table implementation. Fig. 5 shows a bode plot of the acquired models over the complete data set, appendix A gives the calculated transfer functions.

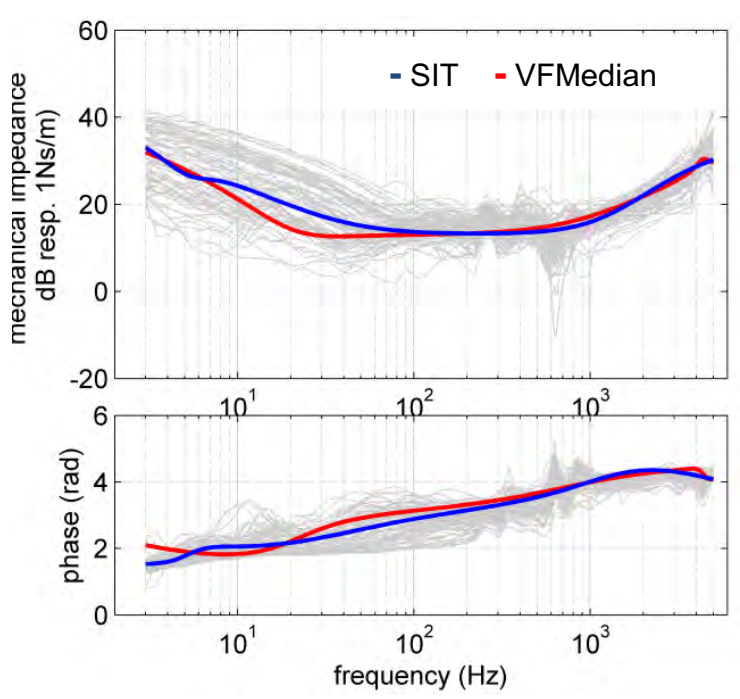

Fig. 5: Bode plot of the acquired models.

For a meaningful interpretation, the calculated transfer functions should be mapped onto the parameter of eq. (4). This is not possible because of an underdetermined system in the case of the system identification toolbox. For the vector fitting method, the resulting term includes a constant term in the denominator that cannot be mapped to the terms in eq. (4). Both methods therefore yield a sufficient approximation of the measurement data, but fail in extracting meaningful parameter values for the network model. 


\section{Conclusion}

The use of a model function and system identification algorithms show the best results in terms of amplitude and phase errors. Commonly known algorithms allow for integration of restrictions and base functions. On several occasions, the results show a shortage of the basic network model for the investigated contact situation. Because of that, no final parameter set could be determined for the network model proposed.

Further work should include more studies on the network topology of the two-port-network for mechanical user impedances. The advantages of network representations are manifold in the design of haptic systems and should justify additional effort in the determination of the models. With improved models, not only the mechanical impedance but also non-linear effects like relaxation could be accounted for. Basis for such advanced models could be distributed networks or combined models based on network parameter and finite element approaches [15].

\section{References}

[1] Meiß, T.; Budelmann, C.; Kern, T.; Sindlinger, S.; Minamisava, C. and Werthschützky, R.: Intravascular palpation and haptic feedback during angioplasty. IEEE Worldhaptics Conference, 2009.

[2] Schlaak, H. F.; Röse, A.; Wohlleber, C.; Kassner, S. and Werthschützky, R.: A Novel Laparoscopic Instrument with Multiple Degrees of Freedom and Intuitive Control. Conference of the International Federation for Medical and Biological Engineering, 2008.

[3] Kassner, S.: Haptische Mensch-MaschineSchnittstelle für ein laparoskopisches ChirurgieSystem. Dissertation, Technische Universität Darmstadt, 2013.

[4] Kern, T. A. (Ed.) Engineering Haptic Devices, Springer, Heidelberg, 2009.

[5] Lenk, A.; Ballas, R.; Werthschützky, R. and Pfeifer, G.: Electromechanical Systems in
Microtechnology and Mechatronics. Springer, Heidelberg, 2011.

[6] Opitz, T., Neupert, C. and Werthschützky, R.: Development of a Haptic Assistive System for Force Transmission onto a Guide Wire. Actuator Conference, Bremen, 2012.

[7] Hogan, N.: Controlling Impedance at the Man/Machine Interface. In: IEEE International Conference on Robotics and Automation (ICRA). Scottsdale, AZ, USA, 1989, pp. 1626-1631.

[8] Hajian, A. and Howe, R.: Identification of the Mechanical Impedance at the Human Finger Tip In: Journal of Biomechanical Engineering, 1997, 119, pp. 109-114.

[9] Hannaford, B. and Anderson, R.: Experimental and Simulation Studies of Hard Contact in Force Reflecting Teleoperation. In: IEEE International Conference on Robotics and Automation. Philadelphia, PA, USA, 1988, S. 584 -589.

[10] Oguztoreli, M. and Stein, R.: Optimal Task Performance of Antagonistic Muscles. In: Biological Cybernetics, 1990, 64, pp. 87-94.

[11] Greenspan, J. and Bolanowski, S.: The Psychophysics of Tactile Perception and Its Peripheral Physiological Basis. In: Kruger, L. et al. (Eds.): Pain and Touch. Academic Press, 1996.

[12] Deschrijver, D., Mrozowski, M., Dhaene, T. and De Zutter, D.: Macromodeling of Multiport Systems Using a Fast Implementation of the Vector Fitting Method, IEEE Microwave and Wireless Components Letters, 2008, 18 (6), pp. 383-385.

[13] Gustavsen, B. and Semlyen A.: Rational approximation of frequency domain responses by Vector Fitting, IEEE Trans. Power Delivery, 1999, 14 (3), pp. 1052-1061.

[14] Gustavsen, B.: Improving the pole relocating properties of vector fitting, IEEE Trans. Power Delivery, 2006, 21(3), pp. 1587-1592.

[15] Starke, E.: Kombinierte Simulation - eine weitere Methode zur Optimierung elektromechanischer Systeme. Dissertation, Technische Universität Dresden, 2009.

\section{Appendix A: model functions}

$\underline{Z}_{\text {user }}(\mathrm{s})$ based on SIT fitting

$$
z_{\text {user }}(s)=\frac{F(s)}{v(s)}=\frac{-52,64 s^{5}-6,44 \cdot 10^{5} s^{4}-2,85 \cdot 10^{9} s^{3}-7,24 \cdot 10^{11} s^{2}-1,89 \cdot 10^{13} s-7,27 \cdot 10^{14}}{s^{5}+7,04 \cdot 10^{4} s^{4}+5,97 \cdot 10^{8} s^{3}+2,23 \cdot 10^{10} s^{2}+7,54 \cdot 10^{11} s}
$$

$\underline{Z}_{\text {user }}(\mathrm{S})$ based on VFMedian fitting

$$
\begin{aligned}
& z_{\text {user }}(s)=\frac{F(s)}{v(s)} \\
& =\frac{-97,72 s^{5}-\left(1,21 \cdot 10^{6}-j 1,24 \cdot 10^{-10}\right) s^{4}-\left(8,01 \cdot 10^{10}-j 9,44 \cdot 10^{-6}\right) s^{3}-\left(3,91 \cdot 10^{14}-j 0,14\right) s^{2}-\left(6,88 \cdot 10^{16}+j 684\right) s-\left(6,71 \cdot 10^{18}+j 1,67 \cdot 10^{5}\right)}{s^{5}+1,14 \cdot 10^{5} s^{4}+1,36 \cdot 10^{9} s^{3}+8,37 \cdot 10^{13} s^{2}+8,5 \cdot 10^{15} s+8,51 \cdot 10^{16}}
\end{aligned}
$$

